\title{
Antagonistic pleiotropy and the stress theory of aging
}

\author{
Peter A. Parsons
}

Received: 8 March 2007/Accepted: 15 May 2007/Published online: 4 July 2007

(C) Springer Science+Business Media B.V. 2007

\begin{abstract}
The ecological stress theory of aging incorporates the normally harsh environments of natural populations and hence restricted resources. Especially towards lethal extremes, positive associations are expected among fitness traits underlain by selection for energetic efficiency favoring genotypes for stress resistance. Positive pleiotropy is therefore expected for fitness traits across varying ages under these conditions. Furthermore, hormetic zones are regions of maximum energetic efficiency, also implying positive pleiotropy. Negative pleiotropy may therefore be mainly a phenomenon of benign environments.
\end{abstract}

Keywords Aging - Antagonistic pleiotropy · Competition · Ecological stress · Energetic efficiency · Fitness $\cdot$ Hormesis $\cdot$ Longevity $\cdot$ Survival

\section{Introduction: From the ecological stress theory of aging to fitness associations}

Maximum reproductive and survival rates are unlikely in free-living animal and in most human

P. A. Parsons

La Trobe University, Bundoora, VIC 3083, Australia

P. A. Parsons $(\square)$

PO Box 906, Unley, SA 5061, Australia

e-mail: pparsons@internode.on.net populations because of exposure to various abiotic (physical) stresses interacting with lesser more transient biotic variables. Consequently organisms struggle to exist in harsh environments that are nutritionally and energetically inadequate, so that survival during aging is mainly determined by the energetic efficiency and metabolic stability required to counter the accumulated stresses of a lifetime. The aging process can therefore be reduced to the energy costs of the internal and external stresses to which organisms are exposed. Well-documented internal stresses can be expressed in terms of oxidative stress, which has a central role in biochemical networks and the metabolic evolution of life on Earth. Furthermore, external or ecological stresses (e.g. aridity, high temperature, solar radiation) affecting aging are being increasingly interpreted in terms of oxidative stress responses. The ecological stress theory of aging assumes that external (ecological) stresses are an integral component of the totality of stresses to which organism are exposed. Under this theory, genetically stress-resistant individuals should accumulate with age; frailer, less robust, less energetically efficient, and more stress-sensitive individuals succumb in parallel. That is, a process of genetic sorting occurs determined by the accumulating responses to internal and external stresses over time, so that energeticallyefficient stress-resistant individuals survive longest.

Fitness approximates to high energetic efficiency and metabolic stability under this extreme environmental model. Positive associations among diverse 
fitness or life-history traits are expected, controlled by stress resistant 'good genes'. Similarly, heterozygotes tend to show higher energetic efficiency than do corresponding homozygotes especially under extreme environments, and therefore should give parallel expectations for associations among lifehistory traits. Energy budgets taking into account the energy cost of environments thus underwrite these predictions for life histories in natural populations. The ecological stress theory of aging is a manifestation of this scenario. Under the stressful scenario pertaining in free-living populations, high energetic and metabolic efficiency should therefore be associated with high (electrophoretic) heterozygosity, high stress resistance, survival, vitality, vigor and resilience, high homeostasis and metabolic stability, low fluctuating asymmetry, rapid development, high mating success, and improved survivorship which normally translates into a long lifespan. The expectation of positive associations among these fitness traits contrasts with more conflicting results obtained under more benign conditions where tradeoffs can more readily develop, which is a complication now to be considered (Parsons 2005, 2007a, b).

\section{Antagonistic pleiotropy?}

Antagonistic pleiotropy, whereby senescence occurs under circumstances where the selection of traits with positive fitness effects early in life changes to negative later in life (Williams 1957), is not possible under the model above. Furthermore, in animal populations subjected to a world full of predators, parasites, various abiotic stresses, food shortages and hence nutritional inadequacy, lifespans are likely to be severely restricted precluding survival to old age. The occurrence of antagonistic pleiotropy would therefore be unlikely in the wild on ecological grounds. Under these conditions, the genetics of survival under environmental hazards becomes predominant (e.g. Jazwinski 2000; Schachter 2000). In other words, the fittest ultimately survive taking into account various environmental constraints, which does not admit a major role for antagonistic pleiotropy. This argument is presented in a preliminary way in Parsons (2002), and will be developed more comprehensively here from a sample of a diffuse literature.
The literature on antagonistic pleiotropy is widespread although many discussions are qualified (e.g. Carey 2003; Guarente 2003). In one discussion, a dependence upon strains of organisms, environmental conditions, and problems in data analysis are mentioned (Arking 1998). Ricklefs and Finch (1995) emphasize how few examples of antagonistic pleiotropy have been established (see also Finch and Kirkwood 2000; Olshanksy and Carnes 2001). Some representative examples of more recent publications follow among the many available reports.

Ostrowski et al (2005) examined mutants of Escherichia coli which increased fitness in glucoselimited laboratory environments for their fitness in five novel resource situations. Most mutants that conferred increased fitness in glucose also conferred increased fitness in the novel resources indicating positive pleiotropy. The few antagonistic pleiotropic effects were primarily restricted to one resource. Similarly, in considering the limits and consequences of adaptive radiation for the availability of glycerol in Pseudomonas fluorescens, Maclean and Bell (2002) found that "mutation accumulation, and not antagonistic pleiotropy was responsible for generating costs of adaptation in this experiment."

Limited evidence for antagonistic pleiotropy has, however, been obtained under controlled and presumably benign laboratory conditions in Drosophila and other species (see Rose 1991; Arking 1998; Carey 2003). For example, Service et al. (1988) presented the results of selection experiments in Drosophila melanogaster designed to distinguish between antagonistic pleiotropy and mutation accumulation as mechanisms important in the evolution of senescence, and concluded that both are important. Laboratory and domesticated populations are normally managed under relatively optimal nutritional conditions, high crowding is avoided, and exposure to extreme abiotic stresses are ameliorated. Furthermore, they may be altered genetically from field conditions especially following periods of laboratory selection. In these contexts, Guarente (2003) discusses mouse strains that are genetically p53; they are virtually cancer-proof but their organs deteriorate at an early age leading to a reduced lifespan. He regards "some sort of devil's bargain between aging and cancer as counterintuitive and unlikely to be correct", since the prevalence of cancer normally increases with age. In any case, what would be the fate of p53 mice in the wild? 
Ultimately, the best evidence for antagonistic pleiotropy may come from well-nourished humans, where environments permit the survival of the old by medical and nutritional interventions. For example, Ricklefs and Finch (1995) discuss a human thrifty genotype which shows antagonistic pleiotropy under the (excessive) nutrition of many populations today. In modern human populations improved nutrition and medicine is occurring, but as a consequence selection for stress resistance is decreasing. The likely consequence is increased survival of relatively stresssensitive and hence relatively unfit individuals, that in the environment of hunter-gatherers would be illequipped for survival.

Capri et al. (2006) summarize evidence for a strong genetic component for survival at extreme ages in humans. Genes that are positively associated with longevity appear to belong mostly to a group of stress-response genes, with the potential to act as modifiers of other genes which have varying metabolic roles. These latter genes, in particular, can have differing biological functions across ages. Capri et al. (2006) therefore conclude that antagonistic pleiotropy can have a major role in humans, where sufficient post-reproductive individuals occur for its detection, although Leroi et al. (2005) caution that very little is known about the early life fitness effects of longevity loci.

It becomes difficult to avoid the conclusion that antagonistic pleiotropy is unlikely to be of consequence under the environmental conditions of natural populations where restricted lifespans preclude most survival to old age. Positive pleiotropy is the likely outcome under these circumstances. Populations under benign environments, where selection for stress resistance is (temporarily) diminished, apparently provide the main exceptions.

\section{The hormetic zone}

Variation in the severity of an environmental stress is expressible on a fitness-stress continuum where the average fitness of individuals relates to the reciprocal of the stress level. Fitness maxima should occur in the benign region of the continuum where abiotic stresses are relatively mild, corresponding to the most likely habitats of organisms (Parsons 2005). For temperature, fitness maxima should occur in regions of some temperature perturbations reflecting those of wild habitats, because a constant temperature or wide fluctuations should be disadvantageous. There is now a substantial and accumulating literature indicating that an array of mild stresses, including cold, heat, hypergravity, physical activity, irradiation, and caloric restriction, can induce longevity extensions in organisms ranging from protozoa, nematodes, flies, rodents and primates. The term hormesis is used to describe such maxima, especially in the toxicology literature (e.g. Luckey 1991; Calabrese and Baldwin 2003).

Adaptation to the mild stresses of the hormetic zone is an evolutionary expectation (Parsons 1990), since all life on Earth is exposed to some stress. Maximum fitness and energetic efficiency therefore should occur in response to exposure to hormeticlevel stresses. Common metabolic responses to hormetic agents include variations in heat shock proteins and especially Reactive Oxygen Species, ROS. Oxidative-stress damage which should be minimal in hormetic zones, can be expressed as an imbalance between free-radical production and antioxidant production. Some cross-protection among hormetic agents is implied because of the general importance of oxidative stress, which may extend the range of hormetic zones for individual stresses (Parsons 2007a). In any case, since the hormetic zone is a region of maximum energetic efficiency, positive associations among fitness traits are expected precluding antagonistic pleiotropy.

Smith Sonneborn (2005) argues that there are hormetic or stress genes induced at threshold levels of physical and chemical environmental agents that provide tolerance to these otherwise harmful agents. These genes activate protective effects in both early and late life. Therefore natural selection of genes critical for early survival can also be positive for extended longevity in older age. Such positive pleiotropy is incompatible with antagonistic pleiotropy since the protective effects occur irrespective of age.

\section{Concluding remarks}

Antagonistic pleiotropy appears most likely under benign environments not normally occurring in natural populations. The common features of such 
environments include reduced severity of abiotic extremes and increased resource availability, implying a lesser restrictive energy status compared with natural populations. Reversion to the environments of natural populations would immediately threaten such populations, and hence any antagonistic pleiotropy relationships.

However, the lack of well-documented field examples remains limiting for conclusions. The extreme-stress-environment model adopted here and in earlier papers allows predictions of associations among fitness traits. Under less restrictive conditions, Reznick et al. (2004) found negative associations in guppies based upon mortalities from high and low predation localities. Mainland and environmentally more benign island opossum populations from Georgia similarly provide comparative data (Austad 1993) warranting more detailed investigations across fitness traits. Outlier populations of $D$. melanogaster where abiotic stresses are readily assessable are useful in this regard for combined laboratory/field investigations (Parsons 2007a).

The development of competitive relationships appears likely to be limited to rather benign environments where abiotic variations are dampened down, so that the energy cost of accommodating stress is relatively low. Therefore, while the evidence for competition among organisms is substantial, it is unlikely to persist in the longer term in natural populations. In any case, the heritability of stressresistance traits can be very high at extremes implying strong selection, while that of competitive ability approaches zero. The long-term evolutionary significance of competition should therefore be low, and perhaps best viewed as second-order effects with the potential to modify the energy costs of abiotic stresses (Parsons 1996, 2005).

Both antagonistic pleiotropy and competition therefore appear to be phenomena mainly manifested under resource and hence energy availability levels not normally found in natural populations. Studies under artificial conditions may therefore be of limited relevance for an understanding of such evolutionary processes in the wild (Kruuk 2004). It is important, therefore, to consider the various evolutionary aging theories put forward over the years in terms of realistic environmental inputs. Here, antagonistic pleiotropy is shown to be an illustrative case study of this central requirement.
Acknowledgement I am grateful to the reviewers for their perceptive analyses, which have substantially improved the paper.

\section{References}

Austad SN (1993) Retarded senescence in an insular population of Virginia opossums (Didelphis virginiana). J Zool London 229:695-708

Arking R (1998) Biology of aging: Observations and principles 2nd edn. Sinauer Associates, Sunderland, Massachusetts

Calabrese EJ, Baldwin LA (2003) Toxicology rethinks its central belief. Nature 421:691-692

Capri M, Salvioli S, Sevini F et al (2006) The genetics of human longevity. Ann NY Acad Sci 1067:252-263

Carey JR (2003) Longevity: The biology and demography of life span. Princeton Univ Press, Princeton

Finch CE, Kirkwood TBL (2000) Chance, development and aging. Oxford Univ Press, New York

Guarente L (2003) Ageless quest. Cold Spring Harbor Press, New York

Jazwinski SM (2000) Metabolic control and ageing. TIG 16:506-511

Kruuk LEB (2004) Estimating genetic parameters in natural populations using the animal model. Phil Trans Roy Soc Lond B 359:873-890

Leroi AM, Bartke A, De Benedictis G (2005) What evidence is there for the existence of individual genes with antagonistic pleiotropic effects? Mech Ageing Dev 126:421-429

Luckey TD (1991) Radiation hormesis. CRC Press, Boca Raton

MacLean RC, Bell G (2002) Experimental adaptive radiation in Pseudomonas. Am Nat 160:569-581

Olshanksy SJ, Carnes BA (2001) The quest for immortality. WW Norton, New York

Ostrowski EA, Rozen DE, Lenski RE (2005) Pleiotropic effects of beneficial mutations in Escherichia coli. Evolution 59:2343-2352

Parsons PA (1990) Radiation hormesis: an evolutionary expectation and the evidence. Appl Radiat Isot 40:857-860

Parsons PA (2002) Life span: does the limit to survival depend upon metabolic efficiency under stress? Biogerontology 3:223-241

Parsons PA (1996) Competition versus abiotic factors in variably stressful environments: evolutionary implications. Oikos 75:129-132

Parsons PA (2005) Environments and evolution: interactions between stress, resource inadequacy and energetic efficiency. Biol Rev 80:589-610

Parsons PA (2007a) The ecological stress theory of aging and hormesis: an energetic evolutionary model. Biogerontology 8:233-242

Parsons PA (2007b) Energetic efficiency under stress underlies positive genetic correlations between longevity and other fitness traits in natural populations. Biogerontology 8:5561

Reznick DN, Bryant MJ, Roff D, Ghalambar CK, Ghalambar DE (2004) Effect of extrinsic mortality on the evolution of senescence in Guppies. Nature 432:1095-1099 
Ricklefs RE, Finch CE (1995) Aging, a natural history. WH Freeman, New York

Rose MR (1991) Evolutionary biology of aging. Oxford Univ Press, New York

Schächter F (2000) Genetics of survival. Ann NY Acad Sci 908:64-70

Service PM, Hutchinson EW, Rose MR (1988) Multiple genetic mechanisms for the evolution of senescence in Drosophila melanogaster. Evolution 42:708-716
Smith Sonneborn J (2005) The myth and reality of reversal of aging by hormesis. Ann NY Acad Sci 1057:165-176

Williams GC (1957) Pleiotropy, natural selection, and the evolution of senescence. Evolution 11:398-411 